\title{
POTENCIAL EVOCADO AUDITIVO DE TRONCO ENCEFÁLICO NO PROGNÓSTICO DO COMA SUPERFICIAL
}

\author{
Brainstem auditory evoked potential response \\ in the prognosis of superficial coma
}

\author{
Libia Camargo Ribeiro Leite ${ }^{(1)}$, Maira de Victor Francisco ${ }^{(2)}$, Sinésio Grace Duarte ${ }^{(3)}$, \\ Cristiane Fregonesi Dutra Garcia ${ }^{(4)}$, Samanta Natália Bizinoto ${ }^{(5)}$
}

\begin{abstract}
RESUMO
O coma é a redução persistente do nível de consciência, arresponsivo a estímulos, devido à baixa atividade cerebral. Para verificar o nível de consciência, um recurso frequentemente utilizado é a Escala de Coma de Glasgow. Outro método que se destaca é o Potencial Evocado Auditivo de Tronco Encefálico, o qual avalia a atividade elétrica das vias auditivas ascendentes, desde o trajeto periférico até o mesencéfalo. O exame é simples, imune a medicamentos depressores e ambientes eletricamente carregados, sendo o mais adequado dos potenciais para a monitoração dos estados de coma. O presente estudo teve por objetivo verificar as características do Potencial Evocado Auditivo de Tronco Encefálico no estado de coma leve (Glasgow 7 - 8) e suas respectivas contribuições. Foi realizado um estudo prospectivo transversal em dois pacientes em coma (Glasgow 7), estado secundário a traumatismo cranioencefálico. Os resultados do exame evidenciaram presença de atividade elétrica em toda extensão da via estudada, em ambos os casos, com indicações de diferentes alterações, quanto à redução na latência entre os intervalos, morfologia e replicação das ondas. Tais diferenças foram contempladas com a evolução de cada caso: caso 1 evoluiu a alta hospitalar e caso 2 evoluiu a óbito. Os resultados confirmaram os achados da literatura, que descreve que a presença do Potencial Evocado Auditivo de Tronco Encefálico normal está associada à boa evolução do caso clínico, enquanto alterações no exame podem sinalizar para um mau prognóstico.
\end{abstract}

DESCRITORES: Coma; Prognóstico; Potenciais Evocados Auditivos

(1) Fonoaudióloga da Prefeitura Municipal de Poços de Caldas - MG; Graduada em Fonoaudiologia pela Universidade de Franca - UNIFRAN, SP, Brasil.

(2) Fonoaudióloga da Prefeitura Municipal de Guaíra, SP; Graduada em Fonoaudiologia pela Universidade de Franca UNIFRAN, SP, Brasil.

(3) Médico; Chefe dos Serviços de Neurocirurgia dos Serviços da Santa Casa de Franca e Hospitais São Joaquim/ Regional; Coordenador/docente do Curso de Medicina UNIFRAN, Franca, SP, Brasil.

(4) Fonoaudióloga; Professora Doutora - Adjunto do Curso de Fonoaudiologia da Faculdade de Medicina da Universidade Federal do Rio de Janeiro - UFRJ, Rio de Janeiro, RJ, Brasil.

(5) Fonoaudióloga da Fundação Santa Casa de Misericórdia de Franca - FSCMF, Franca, SP, Brasil; Aprimoramento em Fonoaudiologia Hospitalar pela Faculdade de Medicina de São José do Rio Preto, SP, Brasil; Aprimoramento em Disfagia e Disfonia pelo CEFAC.

Conflito de interesses: inexistente

\section{INTRODUÇÃO}

O coma é um estado no qual a consciência está reduzida a ponto do indivíduo não despertar mesmo com fortes estímulos sensitivos que deveriam fazê-lo. Portanto o coma pode ser definido como o estado em que o indivíduo não tem conhecimento de si próprio e do ambiente, com ausência ou extrema diminuição do nível de consciência. Este estado de inconsciência é variável em intensidade e está diretamente relacionado à extensão ou estrutura do sistema nervoso central acometida. Sua causa é a lesão ou disfunção do sistema ativador reticular ascendente (SARA), do córtex cerebral difusamente ou de ambos. Inúmeras patologias podem levar o indivíduo ao coma, como os distúrbios metabólicos, os traumatismos cranianos, as infecções do sistema 
nervoso (meningites), intoxicações e a hipertensão intracraniana, entre outras ${ }^{1-3}$.

O nível de consciência pode ser verificado pelo exame neurológico e alguns parâmetros quantificados na chamada Escala de Coma de Glasgow (ECG), sendo eles: abertura ocular, reação motora e resposta verbal, que podem ser obtidos por vários estímulos, desde a atividade espontânea e estímulos verbais até estímulos dolorosos (Tabela 1). As vantagens desta escala são a facilidade da avaliação, do registro e seu uso universal, que favorece a padronização da linguagem referente aos níveis do coma ${ }^{2-5}$.

Tabela 1 - Escala de coma de Glasgow

\begin{tabular}{ccc}
\hline Parâmetros & Resposta Observada & Pontuação \\
\hline \multirow{3}{*}{ Abertura Ocular } & Espontânea & 4 \\
& Com estímulo verbal & 3 \\
& Com estímulo doloroso & 2 \\
& Nenhuma & 1 \\
\hline \multirow{3}{*}{ Melhor Resposta Verbal } & Orientado & 5 \\
& Confuso & 4 \\
& Palavras impróprias & 3 \\
& Sons incompreensíveis & 2 \\
& Nenhuma & 1 \\
\hline \multirow{3}{*}{ Melhor Resposta Motora } & Obedece aos comandos & 6 \\
& Localiza e retira o estímulo & 5 \\
& Localiza o estímulo & 4 \\
& Responde em flexão & 3 \\
& Responde em extensão & 2 \\
\hline
\end{tabular}

Fonte: Koizumi e Araújo (2005, p. 142) ${ }^{5 .}$

O escore é variável entre três e quinze, sendo estes valores representativos do coma grave (menores valores) estado de alerta pleno (maiores valores). Intervalos intermediários indicam os níveis de coma, ou seja, valores entre três e quatro, coma profundo, entre cinco e seis, coma moderado, entre sete e oito, coma leve ${ }^{5}$.

Além do exame neurológico, a utilização de um recurso objetivo na avaliação do tronco encefálico, onde está localizado o SARA, pode oferecer informações significantes a respeito da função da referida estrutura. Dados que o Potencial Evocado Auditivo de Tronco Encefálico (PEATE) pode fornecer, pois consiste no registro da resposta a estímulos auditivos, que geram um gráfico em forma de ondas. Tal exame apresenta sete ondas que aparecem nos primeiros dez milissegundos (mseg) após a apresentação do estímulo auditivo. Cada onda tem diferentes locais de origem: onda I porção distal ao tronco encefálico do nervo auditivo; II - porção proximal ao tronco encefálico do nervo auditivo; III - núcleo coclear; IV - complexo olivar superior; V - lemnisco lateral; VI - colículo inferior e VII - corpo geniculado medial. Dentre elas, as ondas I, III e V são as que oferecem os parâmetros mais importantes para a interpretação do PEATE ${ }^{6-8}$. Essas informações indicam a atividade eletrofisiológica do sistema auditivo, monitoram as sinapses das vias auditivas desde o nervo auditivo, passam por todo o segmento do tronco encefálico, responsável pela maioria das funções vitais do organismo, até o colículo inferior no nível do mesencéfalo. 0 traçado deste potencial é analisado pela morfologia, a replicação, a latência e amplitude das ondas ${ }^{\text {9-11. }}$.

O exame do PEATE, por ser simples, rápido, objetivo, não invasivo, imune a medicamentos depressores e ambientes eletricamente carregados, como é comum nas Unidades de Terapia Intensiva (UTI), torna-se o mais adequado dos potenciais para a monitoração dos estados de coma e no diagnóstico de morte encefálica, como auxiliar para a doação de órgãos ${ }^{12}$. A aplicabilidade do PEATE em situações de internação na UTI vem aumentando por ser um exame de alta confiabilidade e reprodutibilidade ${ }^{7,9-11,13,14}$. 
O presente estudo tem como objetivo verificar as contribuições do uso dos Potenciais Evocados Auditivos de Tronco Encefálico no estado de coma leve (Glasgow $7-8)$, com apresentação de casos clínicos.

\section{APRESENTAÇÃO DOS CASOS}

Inicialmente foi encaminhado o projeto de pesquisa ao Comitê de Ética em Pesquisa da Santa Casa de Misericórdia de Franca, em 01 de junho de 2011, o qual foi aprovado sob o número de protocolo 103/201. Para os casos inclusos no estudo, foi feito contato com os familiares ou responsáveis pelos pacientes, sendo a eles expostos os objetivos do estudo, os procedimentos a serem realizados, seus riscos e benefícios e assim, obtida a autorização para a inclusão no estudo, por meio da assinatura do Termo de Consentimento Livre e Esclarecido, conforme a resolução 196/96.

Foi realizado um estudo do tipo prospectivo transversal, com pacientes comatosos, na UTI da Santa Casa de Misericórdia de Franca, no período de 13 a 30 de setembro de 2011.

Foram considerados os seguintes critérios de inclusão e exclusão dos pacientes no estudo:

Critérios de Inclusão:

- Diagnóstico clínico de coma com escore 3 - 8, segundo a ECG;

- Sem alterações na inspeção do meato acústico;

- Concordância da família ou responsável em participar do estudo.

Critérios de exclusão:

- Doenças infecciosas do sistema nervoso central;

- Doenças desmielinizantes, acidente vascular cerebral de tronco encefálico, tumores localizados próximo ao nervo auditivo ou ao longo do trato auditivo;

- Perdas auditivas relatadas ou outras patologias que pudessem comprometer a presença das ondas do PEATE;

- Artefato e ruído de fundo que excedesse a $10 \%$ dos estímulos apresentados;

- As informações sobre o paciente foram obtidas por meio de consulta aos prontuários e aos familiares.

Para a realização do procedimento foram utilizados os seguintes materiais:
- Equipamento Biologic versão 5.70, modelo 317, dois canais, acoplado a computador convencional, instalado em espaço próprio;

- Otoscópio HEINE mini 2000 e espéculos de diferentes tamanhos;

- Eletrodos de superfície e material para fixação dos mesmos;

- Equipamento de proteção individual (luvas, máscaras, avental);

- Material para anotação dos dados.

Seguindo tais critérios foram selecionados e submetidos ao teste, dois pacientes, sendo ambos do gênero masculino.

\section{Caso 1}

Indivíduo com vinte anos e dois meses de idade, em coma há seis dias, secundário a traumatismo cranioencefálico (TCE), devido a acidente motociclístico. Seu escore na ECG foi 7 desde a internação até o dia da realização do exame.

\section{Caso 2}

Indivíduo com trinta e oito anos e nove meses de idade, internado há vinte dias devido a traumatismo cranioencefálico seguido de coma, após sofrer espancamento. Na admissão apresentava Glasgow 6 e por ocasião da avaliação, G7.

Inicialmente $\mathrm{o}$ paciente era estabilizado $\mathrm{e}$ conduzido em cama-maca, para uma sala de exames dentro da própria UTI, devidamente monitorado. Foram executadas as seguintes etapas:

- Identificação do paciente e seus dados pessoais (idade, gênero, etiologia do coma, tempo de evolução do estado do coma);

- Os pacientes encontravam-se normotérmicos (36-37으).

- Inspeção do meato acústico externo (MAE);

- Preparação dos locais de fixação dos eletrodos (limpeza e colocação da pasta condutora);

- Fixação dos eletrodos: eletrodo ativo (positivo) posicionado na fronte alta, eletrodo referência (negativo) posicionado no lóbulo da orelha testada e eletrodo terra (neutro) colocado no lóbulo da orelha contralateral;

- Conexão ao equipamento;

- Registro das ondas do PEATE para análise, na intensidade de 90 dBNA (decibel Nível de Audição);

- Remoção dos eletrodos do paciente e condução do mesmo de volta ao seu leito na UTI.

- Parâmetros usados na aquisição dos PEA de curta latência (Tabela 3). 
Tabela 2 - Parâmetros para captação do PEATE

\begin{tabular}{|c|c|c|}
\hline & Tipo & Clique, clique filtrado $(1 \mathrm{KHz})-100 \mu$ s tone pip ou tone burst $(500 \mathrm{~Hz})$ \\
\hline \multirow[t]{6}{*}{ Estímulo } & Intensidade & $\begin{array}{l}.70 \text { a } 90 \text { dBNA (integridade SNAC) } \\
\text {. variável (estimação do limiar) }\end{array}$ \\
\hline & Taxa de & 20/s (integridade nervo acústico e das vias auditivas) \\
\hline & $\begin{array}{l}\text { apresentaçao } \\
\text { (rate) }\end{array}$ & .50/s (estimação do limiar - várias intensidades) \\
\hline & Polaridade & Rarefeita \\
\hline & & Ativo: vértex $(\mathrm{Cz})$ ou fronte $(\mathrm{Fpz})$ \\
\hline & Eletrodos & Referência: orelha/mastóide $(\mathrm{A} 1=$ esquerda, $\mathrm{A} 2=$ direita $)$ \\
\hline \multirow[t]{6}{*}{ Aquisição } & & Terra: fronte ou orelha/mastóide contralateral \\
\hline & Registro & Ipsi e contralateral \\
\hline & $\begin{array}{l}\text { Tempo de } \\
\text { análise (janela) }\end{array}$ & $\begin{array}{l}\text { Adultos }=10 \text { a } 15 \mathrm{~ms} \\
\text { Crianças }=15 \text { a } 20 \mathrm{~ms}\end{array}$ \\
\hline & Filtro & $30-3000 \mathrm{~Hz}$ \\
\hline & Amostra & 1000 a 2000 (com replicação) \\
\hline & Sensibilidade & 10 a $20 \mathrm{Mv}$ \\
\hline Paciente & Estado & Relaxado ou dormindo \\
\hline
\end{tabular}

Fonte: Katz, J (2008) ${ }^{6}$

Todos os procedimentos foram executados dentro da UTI.

$\mathrm{Na}$ análise do exame, foram considerados os aspectos relacionados à morfologia do traçado, ou seja, presença das ondas I, III e V; à replicação das ondas, para eliminar a variabilidade e subjetividade de interpretações; à latência absoluta de respostas de cada onda $(I, I I I, V)$ e à latência relativa entre os intervalos interpicos (I-III, III-V, I-V) dentro dos critérios descritos por Hall15 (Tabela 3), conforme referência comumente utilizada na prática clínica; à relação ipsilateral das ondas I-V, em que a amplitude da onda $\mathrm{V}$ deve ser maior que a onda I; e à comparação interaural das ondas $\mathrm{V}$,com diferença máxima de 0,3 mseg, com base na proposta de Figueiredo e Castro Júnior $(2003)^{16}$.

Tabela 3 - Valores de latência das ondas I, III, V e seus intervalos I-III, III-V, I-V

\begin{tabular}{lll}
\hline Ondas & Correspondente (provável) & Latência-adultos (mseg) \\
\hline I & Porção distal ao TE do nervo auditivo & 1,5 a 1,9 \\
II & Porção proximal ao TE do nervo auditivo & 2,5 a 3,0 \\
III & Núcleo coclear & 2,5 a 4,1 \\
IV & Complexo olivar superior & 4,3 a 5,2 \\
V & Lemnisco lateral & 5,0 a 5,9 \\
VI & Colículo inferior & \\
VII & Corpo geniculado medial & \\
\hline INTERPICOS & I - III & 2,14 \\
& III - V & 1,89 \\
& I-V & 4,02 \\
\hline
\end{tabular}

Fonte: Hall III JW (1992) ${ }^{13}$ 


\section{RESULTADOS}

Os resultados do PEATE evidenciaram presença de atividade elétrica em toda extensão da via estudada em ambos os casos, com indicações de diferentes alterações quanto à redução na latência interpicos, à morfologia e replicação das ondas. Tais diferenças foram contempladas com a evolução de cada caso.

\section{Caso 1:}

- Morfologia adequada para o registro A1 (orelha esquerda) e alterada para o registro A4 (orelha direita);
- Replicação adequada para ambos os registros sendo A2 para orelha esquerda e A5 para orelha esquerda;

- Latência Absoluta das ondas I, III, V com valores adequados para ambas as orelhas;

- Latência interpico dos intervalos I-III, III-V, I-V com valores adequados para ambas as orelhas;

- Onda I com amplitude maior que onda V para ambos os registros, o que pode indicar comprometimento no processamento auditivo ${ }^{13}$;

- Comparação interaural das ondas V com valor de 0,20 mseg, o que não indica comprometimento retrococlear.

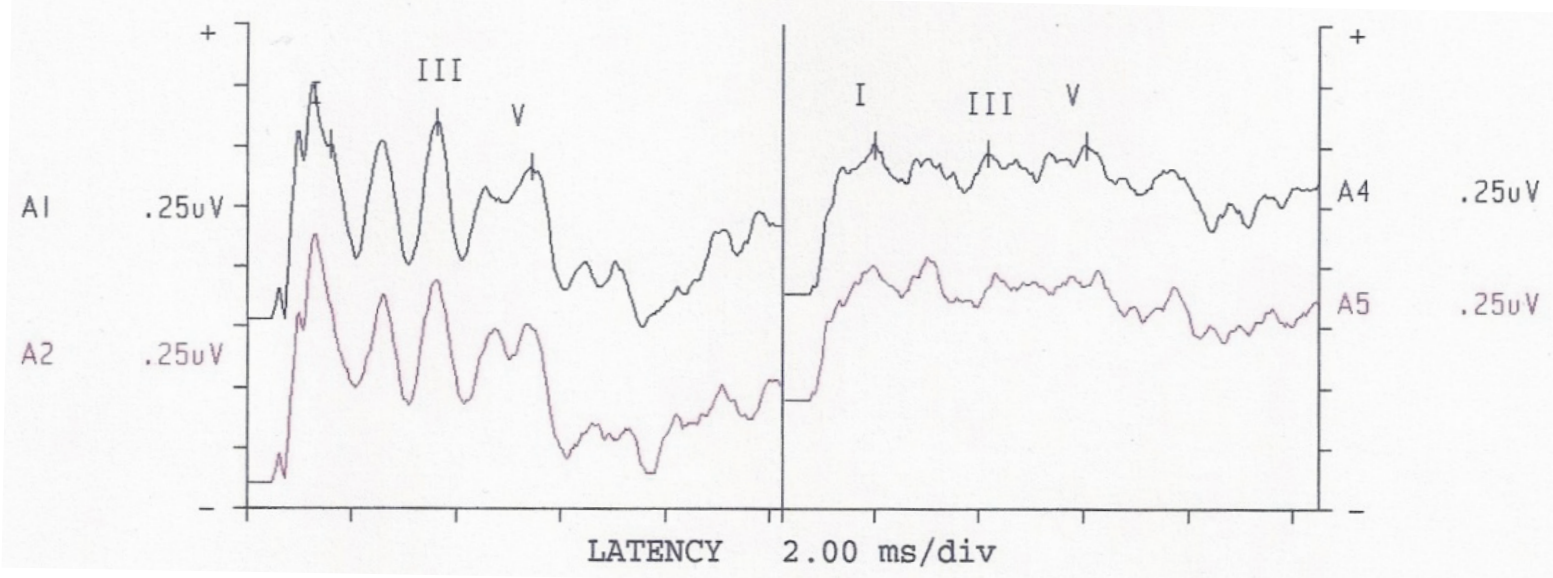

Figura 1 - Registro do PEATE - Caso 1

O resultado do exame indicou atividade de condução do estímulo elétrico preservada na extensão da via auditiva avaliada, ou seja, segmento periférico e tronco encefálico.

O traçado do exame indicou a preservação da função das vias auditivas no tronco encefálico sinalizando a integridade do SARA e, consequentemente, melhor prognóstico, confirmado por reavaliação clínica após vinte dias, quando o paciente encontrava-se na enfermaria e em vias de alta hospitalar, com escore de 14 na ECG.

\section{Caso 2:}

- Morfologia alterada, sendo o registro A6 (orelha direita) melhor que A3 (orelha esquerda);
- Replicação alterada para ambos os registros, sendo A4 para orelha esquerda e A8 para orelha direita;

- Latência absoluta das ondas I, III, V, com valores adequados para ambos os registros;

- Latência interpico dos intervalos I-III, III-V, I-V, com valores alterados para ambos os registros;

- Amplitude da onda I maior que onda V para ambos os registros;

- Comparação interaural das ondas V com valor de 0,16 mseg, o que não indica comprometimento retrococlear.

O resultado do exame indicou atividade de condução do estímulo elétrico preservada, porém comprometida, conforme descrição acima. Estes achados sugerem pior prognóstico, confirmado pelo óbito do paciente. 


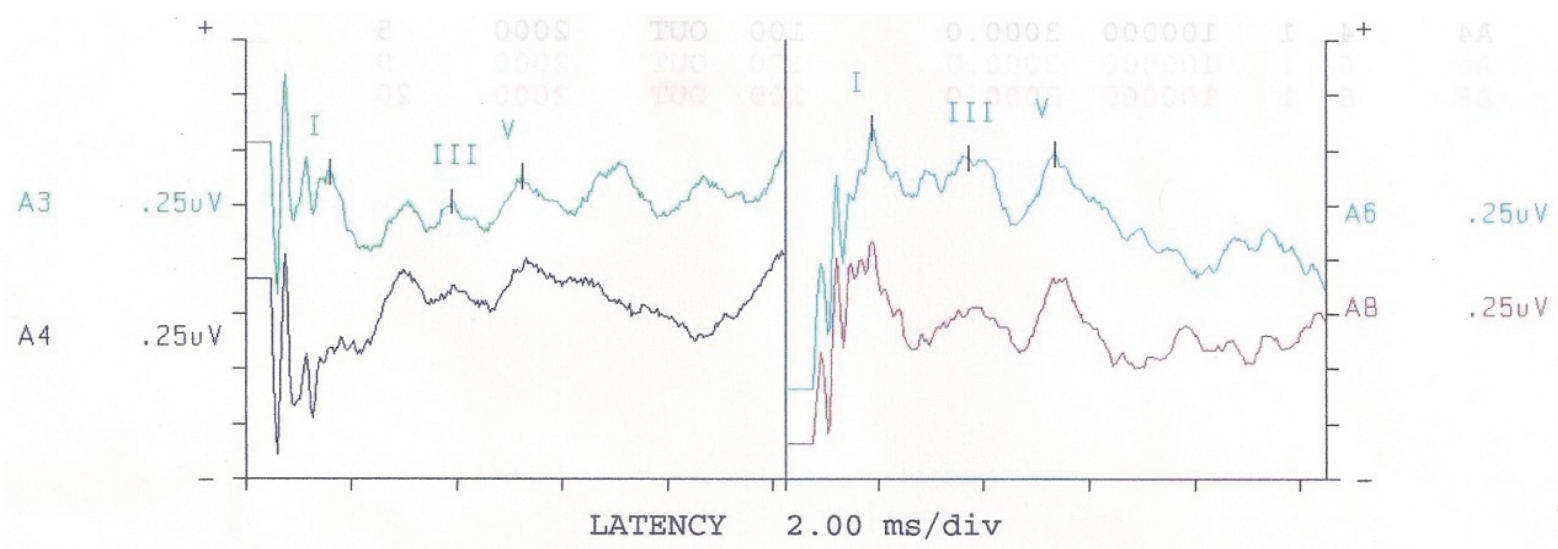

Figura 2 - Registro do PEATE - Caso 2

\section{DISCUSSÃO}

O PEATE se destaca por ser um método competente na aferição do perfil eletrofisiológico do tronco encefálico, em função das vias auditivas ascendentes, que ocupam todo o segmento da referida estrutura do sistema nervoso central, responsável pelas funções do organismo humano, desde as mais simples, como os reflexos primitivos, até reflexos integrados, como os responsáveis pelo ritmo cardíaco, respiração e controle da pressão arterial $^{7,11,12}$.

A deterioração da função do sistema nervoso, na maioria das vezes, se processa de maneira rostro-caudal, ou seja, inicia-se primeiro pelo córtex, as regiões subcorticais e finalmente, o tronco encefálico ${ }^{7,11,12}$.

A avaliação da integridade neurofisiológica do tronco encefálico pelo PEATE dá-se pela sincronia do elemento neural, que pode ser observada pela sobreposição das ondas, morfologia adequada, latência das ondas e intervalos interpicos em indivíduos normais ${ }^{10,12,17 .}$

A literatura apresenta relatos do PEATE como um método eficiente na monitorização dos estados do coma, que contribui para a avaliação clinica e para o prognóstico, além de diagnóstico de morte encefálica, por ser um exame objetivo, não invasivo, de alta confiabilidade, fidedigno, imune a medicamentos depressores do sistema nervoso central e de boa reprodutibilidade, mesmo em ambiente como as UTI(s), eletricamente carregado 10,11,13-15.

$\mathrm{Na}$ presente pesquisa, foram encontrados alguns trabalhos que confirmaram a efetividade do PEATE no prognóstico do coma. Alguns autores verificaram a existência de uma clara relação entre as alterações dos traçados das ondas do PEATE com o nível de disfunção ocorrido no Sistema
Nervoso Central. Outros autores confirmaram a mesma proposta em seus estudos ${ }^{15,18-21}$.

Os casos descritos no presente estudo tiveram como etiologia do coma traumatismo cranioencefálico, por acidente motociclístico e por espancamento. Foram verificadas na literatura, que as principais causas que levam um indivíduo ao coma são as intoxicações exógenas, sendo a mais comum o alcoolismo, traumatismo cranioencefálico (TCE) e doenças cardiovasculares ${ }^{22}$.

O traumatismo cranioencefálico, no presente estudo, a causa do coma, é definido como uma agressão ao cérebro, causada por uma força física externa, temporária ou permanente, que pode causar ao indivíduo um estado de diminuição ou alteração da consciência ${ }^{23}$ Entre as principais causas do TCE estão os acidentes automobilísticos $(50 \%)$, as quedas $(21 \%)$, os assaltos e agressões $(12 \%)$, os esportes e a recreação $(10 \%)^{24,25}$. Alguns autores afirmaram ainda, que a proporção em acidentes automobilísticos é de $90 \%$ para motocicletas e de $9 \%$ para os demais veículos ${ }^{26}$.

Estudos comprovaram que os pacientes com morfologia preservada na pesquisa do PEATE (presença das ondas I, III e V), com replicação do registro adequada, com latências absolutas e intervalos interpicos dentro dos padrões de normalidade evoluíram melhor na maioria dos casos e receberam alta hospitalar. Tal dado pode destacar o papel do PEATE como um recurso na monitorização e determinação do prognóstico no coma ${ }^{11-13}$.

O caso 1 não apresentou alterações na análise do exame do PEATE. Foi observada apenas uma alteração do traçado na orelha direita, lado que o paciente colidiu ao cair em seu acidente. $O$ paciente que sofreu traumatismo cranioencefálico pode apresentar alterações decorrentes do comprometimento da via auditiva em nível periférico, orelha externa, média, interna e nervo auditivo, ou 
também, em nível da via auditiva central, alterações que podem ser detectadas pelo exame do PEATE ${ }^{27}$.

No segundo caso, que apresentou morfologia insatisfatória, porém, com presença das ondas I, III e V, com replicação inadequada (pois não se observou sobreposição dos traçados), latência de intervalos interpicos com valores alterados, evidenciaram anormalidade nas vias auditivas em nível do tronco encefálico, assim como, disfunção no referido sistema. Tais achados são sinalizadores de um prognóstico pior, o que ocorreu com o paciente em estudo. Na literatura, em situação semelhante, os pacientes foram a óbito por apresentar também acometimento encefálico ${ }^{11,13}$.

\section{CONCLUSÃO}

No presente estudo, foi possível confirmar a importância da utilização do PEATE como método auxiliar na avaliação do paciente em coma e a relação dos achados do exame com o prognóstico, exemplificados pelos casos descritos, contemplados por seus resultados, em que a presença do PEATE normal foi associada à boa evolução do caso clínico, enquanto alterações do PEATE foram sinalizadoras de mau prognóstico.

No caso 1, constatou-se que a morfologia do traçado do PEATE, pior à direita, quando comparada com o lado esquerdo, foi justificada pelo lado afetado no TCE; porém, a possibilidade em se verificar a integridade das funções auditivas indicou o bom prognóstico, culminando com a alta hospitalar do paciente.

No caso 2, os resultados do exame do PEATE indicaram atividade comprometida, confirmada pelo pior prognóstico, tendo o paciente evoluído a óbito.

\begin{abstract}
The coma is the persistent reduction consciousness level, not responsive to stimuli, due to low brain activity. To check the consciousness level, a feature often used is the Glasgow Coma Scale. Another method that was been showing up is the Brainstem Evoked Response Audiometry, that evaluates the activity of the ascending auditory pathways from the midbrain to the peripheral route. The test is simple, immune to depressant drugs and electrically charged environments, being the most suitable potential for monitoring the coma. The aim of his study was to examine the characterization of Brainstem Evoked Response Audiometry in mild coma (Glasgow 7-8) and its contribution. We conducted a prospective cross-sectional study in two patients in coma (Glasgow 7), state secondary to head trauma. The test results showed the presence of electrical activity on the entire route in both cases studied, with differences changes relative to the decrease in interpeak latency, morphology, and replication of the waves. These differences were contemplated whit the evolution the each case: case 1 was favorable, but the second died. These results confirm the findings in the literature that describes the Brainstem Evoked Response Audiometry normal are associated with good outcome, while abnormal results are poor prognosis flags.
\end{abstract}

KEYWORDS: Coma; Prognosis; Evoked Potentials Auditory

\section{REFERÊNCIAS}

1. Goldman L, Ausiello D. CECIL, Tratado de Medicina Interna. 22 ${ }^{\mathrm{a}}$ ed. Rio de Janeiro: Elsevier; 2005.

2. Bacheschi LA, Nitrini R. A neurologia que todo médico deve saber. $2^{\underline{a}}$ ed. São Paulo: Atheneu; 2008.

3. Greenberg MS. Handboob of Neurosurgery. $7^{\text {a }}$ ed. Tampa, Florida: Thieme; 2010. p. 279.
4. Rabello GD. In: Stávale M. Bases da Terapia Intensiva Neurologica. $2^{\underline{a}}$ ed. São Paulo: Santos; 2011. p. 281

5. Koizumi MS, Araújo GL. Escala de Coma de Glasgow - Subestimação em pacientes com respostas verbais impedidas. Acta Paul. Enferm. 2005;18(2):136-42.

6. Katz, J. Tratado de audiologia clínica. $4^{\underline{a}}$ ed. São Paulo: Manole; 1999. 
7. Musiek FE, Rintelman WF. Perspectivas atuais em avaliação auditiva. São Paulo: Manole; 2001.

8. Møller AR, Janetta PJ. Compound action potentials recorded intracranially from the auditory nerve in man. Exp. Neurol. 1981;74:862-74.

9. Jardim M, Person OC, RapoportPB. Potencial evocado auditivo de tronco encefálico como auxílio diagnóstico de morte encefálica. Pró-Fono R. Atual. Cient. 2008;20(2):123-8.

10. Sousa LCA, Piza MRT, Ferez M, Rodrigues LS, Ruiz DB, Schmidt VB. O BERA como instrumento de avaliação funcional do tronco cerebral em cirurgias com hipotermia profunda e parada circulatória total. Rev. Bras. Otorrinolaringol. 2003;69(5):664-70.

11. Luccas FJC, Lopes JA, Caivano ABS, Lourenço FMR, Silva MMR, Silva MLI. in Stavale M. Bases da Terapia Intensiva Neurologica; $2^{\underline{a}}$ ed. Santos; 2011. p. 431.

12. Souza Jr AA, Cuehuen JAN, Fukuda Y, Almeida JM , Gonçalves YP , Lara R. Audiometria de Tronco Encefálico (ABR) e estadiamento clínico (Glasgow) no diagnóstico de morte encefálica em candidatos à doação de órgãos. Rev. Bras. Ter. Intensiva. 2004;16(2):82-7.

13. Sousa LCA, Piza MRT, Alvarenga KF, Cóser PL. Eletrofisiologia da audição e emissões otoacústicas. $1^{\text {a }}$ ed. São Paulo: Novo Conceito Saúde; 2008. p. 49-82, 349-53.

14. Marseillan RF, Oliveira JAA, Vecchio FD. Audiometria de respostas elétricas do tronco cerebral humano. Rev. Bras. de Otorrinolaringol. 1997;43:229-38.

15. Hall III JW. Handbook of auditory evoked responses. Boston: Allyn and Bacon; 1992.

16. Figueiredo MS, Castro Jr NP de. Potenciais evocados auditivos precoces. In: Campos CAH de, Costa HOO. Tratado de otorrinolaringologia. São Paulo: Roca; 2003. p. 522-9.

17. Sousa LCA, Rodrigues LS, Pizza MRT, Ferreira DR, Ruiz DB. Achado ocasional de doenças neurológicas durante a pesquisa da surdez infantil através do BERA. Rev. Bras. de Otorrinolaringol. 2007;73(3):424-8.

Recebido em: 21/11/2012

Aceito em: 15/04/2013

Endereço para correspondência:

Libia Camargo Ribeiro Leite

Rua: Adornírio Gonçalves nº 173 - Centro

Botelhos - MG - Brasil

CEP: $37720-000$

E-mail: libia_camargo@ @otmail.com

libiacamargo@ymail.com
18. Uziel A, Benezech J. Auditory brain-stem responses in comatose patients: relationship with brain-stem reflexes and levels of coma. Eletroencephalogr. Clin. neurophisiol.1978;45:515-24.

19. Facco E, Munari M, Liviero MC, Caputo P, Martini A, Toffoletto $F$ et al. Serial recordings of auditory brainstem responses in severe head injury: relationship between test timing and prognostic power. Intensive Care Med. 1988;14:422-8.

20. MjoenS, Nordby HK, TorvikA. Auditory evoked brainstem responses (ABR) incoma due to severe head trauma. Acta Otolaryngol. 1983;95:131-8.

21. Tsubokawa $T$, Nishimoto $H$, Yamoto $T$, Kitamura M, Katayama Y, MoriyasuN. Assessment of brainstem damage by the auditory brainstem response in acute severe head injury. Journal of Neurology, Neurosurgery and Psyquiatry. 1980;43:1005-11.

22. Andrade AF de, Carvalho RC, Amorim RLO de, Paiva WS, Figueiredo EG, Teixeira MJ. Coma e outros estados de consciência. Rev Med. 2007;86(3):123-31.

23. Morgado FL, ROSSI LA. Correlação entre a escala de coma de Glasgow e os achados de imagem de tomografia computadorizada em pacientes vítimas de traumatismo cranioencefálico. Radiol Bras. 2011;44(1):35-41.

24. Oliveira SG, Wibelinger LM, Luca RD. Traumatismo Cranioencefálico: uma revisão bibliográfica. [homepage na internet]. Site Fisioweb Wgate; 27 set. 2005 [ Acesso em: 04 out. 2011]; [cerca de 15p.] Disponível em: http://www.wgate. com.br/conteudo/medicinaesaude/fisioterapia/ neuro/traumatismo_tce.htm

25. Carlotti Jr CR, Boullosa JLR, Dias LAA, Oliveira RS, Colli BO. Traumatismos cranioencefálicos. Medicina.1995;28(4):765-76.

26. Koizumi MS. Padrão das lesões nas vítimas de acidentes de motocicleta. Rev. Saúde Pública. 1992;26:306-15.

27. Marangoni AT, Santos RBF, Suriano IC, Ortiz KZ, Gil D. Avaliação eletrofisiológica da audição em indivíduos após traumatismo cranioencefálico. Rev. CEFAC [periódico na Internet] 2013; [acesso em: 25 de março de 2013];15(1):58-68]. Disponível em: http://www.scielo.br/scielo.php?pid=S151618462011005000138\&script=sci_arttext 